\title{
Radionuclide Imaging of Musculoskeletal Infection: A Review
}

\author{
Christopher J. Palestro \\ Department of Radiology, Hofstra Northwell School of Medicine, Hempstead, New York
}

Learning Objectives: On successful completion of this activity, participants should be able to describe (1) the principle of combined labeled leukocyte/marrow
imaging; (2) at least one advantage of ${ }^{18} \mathrm{~F}$-FDG PET or PET/CT over MRI for diagnosing spondylodiskitis; and (3) at least one advantage of SPECT/CT over planar imaging in the diagnosis of pedal osteomyelitis in diabetic patients.

Financial Disclosure: The author of this article has indicated no relevant relationships that could be perceived as a real or apparent conflict of interest.

CME Credit: SNMMI is accredited by the Accreditation Council for Continuing Medical Education (ACCME) to sponsor continuing education for physicians. SNMMI designates each JNM continuing education article for a maximum of 2.0 AMA PRA Category 1 Credits. Physicians should claim only credit commensurate with the extent of their participation in the activity. For CE credit, SAM, and other credit types, participants can access this activity through the SNMMI website (http://www.snmmilearningcenter.org) through September 2019.

There are numerous imaging tests for diagnosing musculoskeletal infection. Radiographs are routinely performed, because even when not diagnostic, they provide an anatomic overview of the region of interest that could influence subsequent procedure selection and interpretation. MRI is sensitive and provides superb anatomic detail. Bone scintigraphy accurately diagnoses osteomyelitis in bones not affected by underlying conditions. ${ }^{67} \mathrm{Ga}$ is used primarily for spondylodiskitis. Although in vitro labeled leukocyte imaging is the radionuclide test of choice for complicating osteomyelitis such as diabetic pedal osteomyelitis and prosthetic joint infection, it is not useful for spondylodiskitis. Antigranulocyte antibodies and antibody fragments have limitations and are not widely available. ${ }^{111}$ In-biotin is useful for spondylodiskitis. Radiolabeled synthetic fragments of the antimicrobial peptide ubiquicidin are promising infection-specific agents. ${ }^{18} \mathrm{~F}-\mathrm{FDG}$ is the radiopharmaceutical of choice for spondylodiskitis. Its role in diabetic pedal osteomyelitis and prosthetic joint infection is not established. Preliminary data suggest ${ }^{68} \mathrm{Ga}$ may be useful in musculoskeletal infection. ${ }^{124}$ I-fialuridine initially showed promise as an infection-specific radiopharmaceutical, but subsequent investigations were disappointing. The development of PET/CT and SPECT/CT imaging systems, which combine anatomic and functional imaging, has revolutionized diagnostic imaging. These hybrid systems are redefining the diagnostic workup of patients with suspected or known infection and inflammation by improving diagnostic accuracy and influencing patient management.

Key Words: FDG; gallium; labeled leukocytes; osteomyelitis; PET/CT; SPECT/CT

J Nucl Med 2016; 57:1406-1412

DOI: 10.2967/jnumed.115.157297

\section{A}

cute osteomyelitis arises hematogenously or via direct inoculation. Local risk factors include open fractures, recent surgery, and orthopedic hardware. Systemic risk factors include diabetes, immunosuppression, and substance abuse (1). The diagnosis is not

Received Feb. 29, 2016; revision accepted Jun. 29, 2016.

For correspondence or reprints contact: Christopher J. Palestro, Division of Nuclear Medicine and Molecular Imaging, Long Island Jewish Medical Center, 270-05 76th Ave., New Hyde Park, NY 11040.

E-mail: palestro@northwell.edu

Published online Jul. 7, 2016.

COPYRIGHT (c) 2016 by the Society of Nuclear Medicine and Molecular Imaging, Inc. always obvious, and imaging procedures are part of the diagnostic workup. There are numerous imaging tests from which to choose, none of which is optimal for every situation. This article reviews the various radiopharmaceuticals used in the diagnostic workup of patients suspected of having musculoskeletal infection.

\section{MORPHOLOGIC IMAGING}

\section{Radiography}

Radiographs are the initial imaging study performed for suspected osteomyelitis. Early findings include soft-tissue swelling and blurring of adjacent fat planes. Medullary trabecular lysis, cortical destruction, and periosteal reaction may not appear for more than a week (Supplemental Fig. 1; supplemental materials are available at http://jnmt.snmjournals.org). Although sensitivity and specificity only range from about $50 \%$ to $75 \%$ and from $75 \%$ to $83 \%$, respectively, radiographs provide an anatomic overview of the areas of interest potentially guiding selection and interpretation of subsequent procedures (2).

\section{MRI}

The earliest findings, which can appear within $2 \mathrm{~d}$ after onset of infection, are a decrease in signal intensity on T1-weighted MRI sequences and an increase in signal intensity on fat-suppressed T2weighted sequences caused by inflammatory marrow edema (Supplemental Fig. 2). Periosteal reaction and adjacent soft-tissue edema appear later. Intravenous contrast material is useful for evaluating abscesses and differentiating synovial fluid from synovial thickening. The test has a very high negative predictive value for excluding osteomyelitis. Its positive predictive value, the ability to differentiate osteomyelitis from noninfectious causes of abnormal marrow signal intensity, is lower (2).

\section{CT and Ultrasonography}

$\mathrm{CT}$ and ultrasonography are not primary imaging modalities for osteomyelitis. On CT, acute osteomyelitis appears as an area of increased density in the medullary cavity, accompanied by blurring of fat planes, periosteal reaction, and cortical loss (2).

Ultrasonography is useful in regions complicated by orthopedic instrumentation and in patients for whom MRI is contraindicated. The diagnosis of acute osteomyelitis is made by identification of a subperiosteal abscess. Before the formation of a subperiosteal abscess, the diagnosis may be missed. Soft-tissue abscesses adjacent to bone may be misinterpreted as subperiosteal abscesses (2). 


\section{RADIONUCLIDE IMAGING}

\section{Bone Scintigraphy}

Bone scintigraphy is performed with ${ }^{99 \mathrm{~m}} \mathrm{Tc}$-labeled diphosphonates. Uptake depends on blood flow and rate of new bone formation. Three-phase bone scintigraphy usually is performed for osteomyelitis and consists of the perfusion phase, followed immediately by the soft-tissue phase. The skeletal phase is performed 2-4 h later. Focal hyperperfusion, focal hyperemia, and focally increased bony uptake is the classic appearance of osteomyelitis. The test is sensitive and specific in otherwise normal bone. Preexisting conditions such as fracture, orthopedic hardware, and adjacent soft-tissue infection decrease specificity (3).

\section{${ }^{67}$ Ga Scintigraphy}

${ }^{67} \mathrm{Ga}$ uptake in infection is multifactorial. Approximately $90 \%$ of the injected ${ }^{67} \mathrm{Ga}$ is in plasma, nearly all of which initially is transferrin-bound. Increased blood flow and vascular membrane permeability result in increased delivery and accumulation of ${ }^{67} \mathrm{Ga}$ at foci of infection. ${ }^{67} \mathrm{Ga}$ binds to lactoferrin, which is present in most infections. Direct bacterial ${ }^{67} \mathrm{Ga}$ uptake has been reported. Siderophores, chelates produced by bacteria, are ${ }^{67} \mathrm{Ga}$-avid. The siderophore-gallium complex presumably is transported into the bacterium and phagocytized by macrophages. Some ${ }^{67} \mathrm{Ga}$ may be transported by circulating leukocytes. Imaging usually is performed $18-72 \mathrm{~h}$ after injection. Currently, ${ }^{67} \mathrm{Ga}$ imaging is used primarily for spondylodiskitis (Fig. 1) (3).

\section{Labeled Leukocyte Scintigraphy}

In Vitro Labeled Leukocytes. ${ }^{111}$ In-oxyquinoline $\left({ }^{111} \mathrm{In}\right)$ and ${ }^{99 \mathrm{~m}}$ Tc-exametazime $\left({ }^{99 \mathrm{~m}} \mathrm{Tc}\right)$ are most often used to label leukocytes in vitro. Leukocyte uptake depends on intact chemotaxis, number and types of cells labeled. and cellular response to a particular stimulus. The circulating leukocyte count should be 2,000 or more per microliter for diagnostically acceptable images. Because most leukocytes labeled are neutrophils, labeled leukocyte imaging is most sensitive for neutrophil-mediated infections. Intense pulmonary activity on images obtained soon after labeled leukocyte infusion is caused by cellular activation during labeling, which impedes passage of labeled leukocytes through the pulmonary vasculature (4).

Advantages of the ${ }^{111}$ In radiolabel include stability; a normal distribution at $24 \mathrm{~h}$ after infusion limited to liver, spleen, and bone marrow; and ability to perform delayed imaging. Complementary bone marrow imaging can be performed before, simultaneously

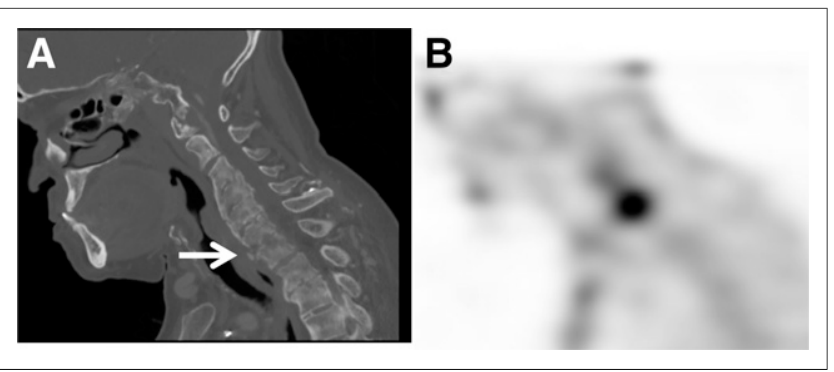

FIGURE 1. Osteomyelitis cervical spine. (A and B) Sagittal CT image (A) shows destructive changes at C6-C7 level (arrow) corresponding to area of intense radiopharmaceutical uptake on sagittal ${ }^{67} \mathrm{Ga}$ SPECT image (B). (Reprinted with permission of (3).) with, or after ${ }^{111}$ In-labeled leukocyte imaging. Disadvantages include limited resolution and the 18- to 30-h delay between injection and imaging (4).

${ }^{99 m}$ Tc-labeled leukocyte distribution is more variable because some of the technetium elutes from the leukocytes and is excreted via the kidneys and hepatobiliary system. Therefore, in addition to reticuloendothelial system visualization, the urinary tract, bowel, and gallbladder often are seen. Higher-resolution images and ability to detect abnormalities shortly after injection are advantages of ${ }^{99 \mathrm{~m}}$ Tc-labeled leukocytes. Disadvantages include label instability and the short half-life of ${ }^{99 \mathrm{~m}} \mathrm{Tc}$, which limits delayed imaging. There should be $2-3 \mathrm{~d}$ between ${ }^{99 \mathrm{~m}} \mathrm{Tc}$-labeled leukocyte imaging and marrow imaging (4).

Leukocytes accumulate in infection and marrow, and it is not always possible to differentiate between them on labeled leukocyte images. ${ }^{99 \mathrm{~m}} \mathrm{Tc}$-sulfur colloid bone marrow imaging facilitates this differentiation. Both radiopharmaceuticals accumulate in marrow; only labeled leukocytes accumulate in infection. Labeled leukocyte/marrow imaging is positive for osteomyelitis when activity is present on the labeled leukocyte image without corresponding activity on the marrow image (Figs. 2 and 3). The accuracy of labeled leukocyte/marrow imaging is approximately $90 \%$ (5). Dualtime-point imaging at 3-4 h and 20-24 h after labeled leukocyte infusion has been suggested as an alternative to labeled leukocyte/ marrow imaging (6).

In Vivo Labeled Leukocytes. Besilesomab, a murine monoclonal IgG1 antibody, binds to cross-reacting antigen-95 on granulocyte and granulocyte precursor cell membranes. About $10 \%$ of ${ }^{99 \mathrm{~m}} \mathrm{Tc}$-besilesomab is neutrophil-bound. Twenty percent circulates freely, localizing in infection through nonspecific mechanisms. The incidence of human antimurine antibody response, which occurs in more than $30 \%$ of patients receiving repeated injections, is a disadvantage (7).

Sulesomab is a $50-\mathrm{kDa}$ fragment antigen binding $\left(\mathrm{Fab}^{\prime}\right)$ portion of an IgG1 class murine monoclonal antibody that binds to normal crossreactive antigen-90 on leukocytes. Approximately 3\%-6\% of the ${ }^{99 \mathrm{~m}} \mathrm{Tc}$-sulesomab injected is associated with circulating neutrophils; by $24 \mathrm{~h}$, about $35 \%$ is in the marrow. Initial investigations suggested that uptake in infection includes binding to circulating neutrophils and to leukocytes present at the site of infection. Subsequent data suggested that accumulation in infection is nonspecific (7).

Interleukin 8 , a chemotactic cytokine that binds to $\alpha$-chemokine receptor types 1 and 2 on leukocytes, rapidly accumulates in infection. Limited investigations suggest that this agent accurately diagnoses musculoskeletal infection (7).

\section{In-Biotin}

Biotin, or vitamin B7, which is important in glucose metabolism, is a bacterial growth factor $(8) .{ }^{111}$ In-biotin, alone and combined with streptavidin, has been used for imaging infection. Advantages include same-day imaging and little or no bone marrow uptake. Antibiotic therapy does not affect sensitivity (7).

\section{Radiolabeled Antibiotics}

Radiolabeled antibiotics were an attempt at developing infectionspecific agents. The most extensively investigated radiolabeled antibiotic is ${ }^{99 \mathrm{~m}} \mathrm{Tc}$-ciprofloxacin. Initial investigations reported high sensitivity and specificity. Subsequent investigations raised serious questions about specificity, and enthusiasm for radiolabeled antibiotics has faded (7). 


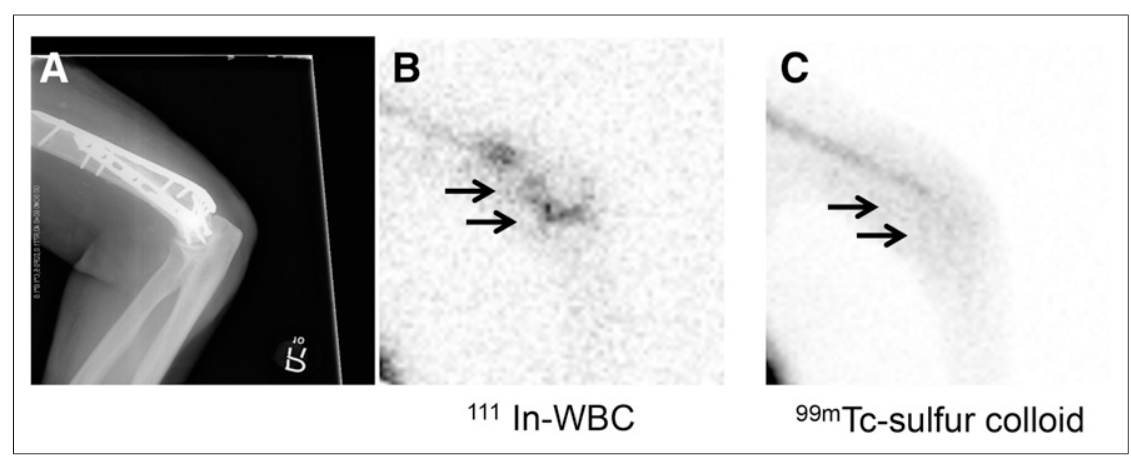

FIGURE 2. Infected right-upper-extremity hardware. (A) Radiograph demonstrates hardware in distal right humerus. ( $B$ and $C$ ) Spatially incongruent distribution of activity (arrows) is seen on ${ }^{111}$ In-labeled leukocyte image (B) and ${ }^{99 m T c}$-sulfur colloid bone marrow image (C).

typically is performed about $1 \mathrm{~h}$ after injection. Uptake usually normalizes within 3-4 mo after trauma or surgery, and degenerative bone changes ordinarily show only mildly increased uptake (3).

\section{${ }^{18}$ F-FDG-Labeled Leukocytes}

In an effort to develop a more specific PET radiopharmaceutical, leukocytes have been labeled in vitro with ${ }^{18}$ F-FDG. Disadvantages include the 110 -min half-life of ${ }^{18} \mathrm{~F}$, which precludes off-site labeling and delayed imaging, a lower and more variable labeling efficiency than that of ${ }^{111} \mathrm{In}$-oxine, and rapid ${ }^{18} \mathrm{~F}-\mathrm{FDG}$ elution from leukocytes (3).

\section{${ }^{68} \mathrm{Ga}-$ Citrate}

Although the imaging characteristics of

\section{Radiolabeled Antimicrobial Peptides}

Antimicrobial peptides, part of the natural defenses of most living organisms, are small, cationic, and amphipathic (hydrophilic and hydrophobic). Their expression may be constant or induced on contact with microbes. They may be transported by circulating leukocytes. Antimicrobial peptides kill microbes but are not harmful to mammalian cells, and their therapeutic and diagnostic potential is being investigated $(7,9,10)$. Radiolabeled synthetic fragments of ubiquicidin, which is present in murine macrophages, have been the most extensively studied antimicrobial peptides $(7,10,11)$. ${ }^{99 \mathrm{~m}}$ Tc-ubiquicidin $29-41$ appears to be sensitive and specific for musculoskeletal infection and has shown promise for monitoring treatment response (7). ${ }^{68} \mathrm{Ga}$-labeled ubiquicidin 29-41 successfully detects bacterial infection (11).

\section{F-FDG}

${ }^{18} \mathrm{~F}-\mathrm{FDG}$ is transported into cells via glucose transporters and is phosphorylated by hexokinase to ${ }^{18} \mathrm{~F}-2{ }^{\prime}-{ }^{18} \mathrm{~F}-\mathrm{FDG}-6$ phosphate but not metabolized further. Uptake by leukocytes depends on cellular metabolic rate and number of glucose transporters. There is an increased number and expression of glucose transporters by activated inflammatory cells and an increased affinity of these transporters for ${ }^{18} \mathrm{~F}$-FDG (3).

${ }^{18} \mathrm{~F}-\mathrm{FDG}$ PET is a relatively high-resolution imaging test that provides precise radiopharmaceutical localization. The small ${ }^{18}$ F-FDG molecule enters poorly perfused areas rapidly. Imaging

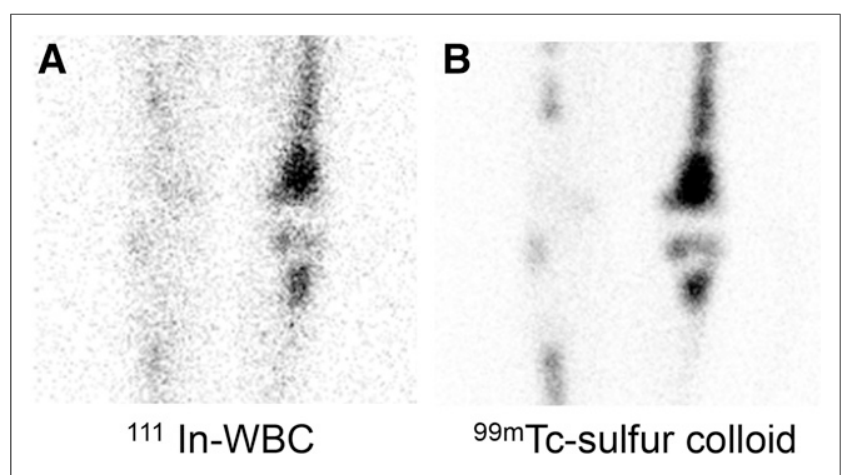

FIGURE 3. Bone marrow expansion. (A) Irregularly increased activity in left lower extremity on ${ }^{111}$ In-labeled leukocyte image from patient with sickle cell disease might be interpreted as osteomyelitis. (B) Distribution of activity on ${ }^{99 \mathrm{~m} T c-s u l f u r}$ colloid bone marrow image, however, is same.
${ }^{68} \mathrm{Ga}$-citrate are superior to those of ${ }^{67} \mathrm{Ga}$, uptake mechanisms are the same. Disadvantages of ${ }^{67} \mathrm{Ga}$, including uptake in inflammation, trauma, and tumor, also apply to ${ }^{68} \mathrm{Ga}$-citrate. Another potential disadvantage is its short half-life (68 min). Complexing ${ }^{68} \mathrm{Ga}$-citrate with peptides may overcome these problems (12).

\section{4|-Fialuridine}

${ }^{124}$ I-fialuridine is a specific substrate of bacterial thymidine kinase. In a pilot study, all 8 patients with musculoskeletal infection demonstrated ${ }^{124}$ I-fialuridine accumulation in the infection. There was no abnormal uptake in the control (13). Results of subsequent investigations, however, have been disappointing $(14,15)$.

\section{INDICATIONS}

No one agent is equally efficacious throughout the skeleton. Selecting the most appropriate study depends on the clinical situation. In adults, it is useful to divide musculoskeletal infections into 3 categories: spine, diabetic foot, and prosthetic joint.

\section{Spondylodiskitis}

Spondylodiskitis arises hematogenously, through direct external inoculation, or through spread from contiguous tissues and can extend into adjacent soft tissues. Hematogenous pyogenic spondylodiskitis most often involves the lumbar spine. Tuberculous infection more commonly affects the thoracic spine and is more likely to involve more than two vertebrae (16).

Radionuclide imaging is a valuable adjunct to MRI for spondylodiskitis. Although bone scintigraphy is used for screening, falsenegative results occur. It is not sensitive for detecting soft-tissue infections that accompany, or mimic, spinal infections. Scan findings may remain abnormal for some time after infection has resolved. ${ }^{67} \mathrm{Ga}$ imaging improves the specificity of, and may detect infection sooner than, bone scintigraphy and identifies accompanying softtissue infections $(3,17,18) .{ }^{67} \mathrm{Ga}$ SPECT/CT reduces false-positive and false-negative results and identifies soft-tissue infection (3).

${ }^{67} \mathrm{Ga}$ has disadvantages. Its physical characteristics and normal distribution can confound image interpretation. Although the test may become positive shortly after injection, imaging typically is performed $18-72 \mathrm{~h}$ after injection. ${ }^{67} \mathrm{Ga}$ accumulates in inflammation, tumor, and trauma, which can coexist with, or mimic, infection.

Labeled leukocyte imaging is not useful for diagnosing spondylodiskitis. Approximately $50 \%$ of cases present as areas of nonspecific decreased activity (4). 
${ }^{111}$ In-biotin accurately diagnoses spondylodiskitis. Performing SPECT/CT accurately differentiates bone from soft-tissue infection and helps guide therapy $(19,20)$.

${ }^{18} \mathrm{~F}-\mathrm{FDG}$ imaging consistently outperforms bone, ${ }^{67} \mathrm{Ga}$, and antigranulocyte antibody imaging and compares favorably with MRI (21-30). Gratz et al. (22) reported that ${ }^{18}$ F-FDG PET was superior to MRI for low-grade spondylitis/diskitis. Stumpe et al. (25) reported that, in patients with lumbar spine vertebral end-plate abnormalities, ${ }^{18}$ F-FDG PET was $100 \%$ sensitive and specific. MRI was $50 \%$ sensitive and $96 \%$ specific. In an investigation of patients with inconclusive conventional imaging results, the sensitivity, specificity, and accuracy of ${ }^{18} \mathrm{~F}-\mathrm{FDG}$ PET/CT were $81.8 \%, 100 \%$, and $89.5 \%$, respectively, versus $75 \%, 71.4 \%$, and $74.1 \%$, respectively, for MRI (26). Fuster et al. (27) compared ${ }^{18}$ F-FDG PET/CT and MRI. Sensitivity and specificity for ${ }^{18}$ F-FDG PET/CT were $83 \%$ and $88 \%$, respectively, versus $94 \%$ and $38 \%$, respectively, for MRI. In patients with brucellar spondylodiskitis, ${ }^{18} \mathrm{~F}-\mathrm{FDG}$ PET/CT identified all foci of infection seen on MRI and revealed additional spinal lesions in 3 patients, as well as new paravertebral soft-tissue involvement and epidural masses. This additional information influenced patient management (29). Nakahara et al. (30) reported that ${ }^{18} \mathrm{~F}$-FDG PET/CT was superior to MRI for localizing sites of infection and guiding minimally invasive surgery.

${ }^{18} \mathrm{~F}-\mathrm{FDG}$ appears useful for monitoring treatment response in spondylodiskitis. Riccio et al. (31) reported that patients with poor treatment response had persistent bone and soft-tissue ${ }^{18} \mathrm{~F}-\mathrm{FDG}$ uptake. ${ }^{18} \mathrm{~F}$-FDG uptake confined to the margins of a destroyed disk after treatment did not indicate infection. Successful treatment of brucellar spondylodiskitis was associated with a significant decrease in ${ }^{18}$ F-FDG uptake (29). Skanjeti et al. (28) reported that ${ }^{18}$ F-FDG PET/CT was more accurate than MRI (90\% vs. $\left.61.5 \%\right)$ for assessing treatment response.

There are some circumstances in which ${ }^{18}$ F-FDG may be less useful. Differentiating infection from tumor and infection superimposed on tumor may be problematic. Significant focal ${ }^{18}$ F-FDG uptake in degenerative spine disease occasionally occurs (3). Foreign body reaction around uninfected spinal implants may also cause increased uptake (Fig. 4) (32). Regardless, published data support ${ }^{18} \mathrm{~F}-\mathrm{FDG}$ imaging as the nuclear medicine test of choice for diagnosing spondylodiskitis, although more data are needed before one can conclude that it should be the initial imaging test for this entity.

Data about ${ }^{68} \mathrm{Ga}$-citrate in spondylodiskitis are limited. Nanni et al. (33) reported that the test was $100 \%$ sensitive and $76 \%$ specific. False-positive results were associated with tumor.

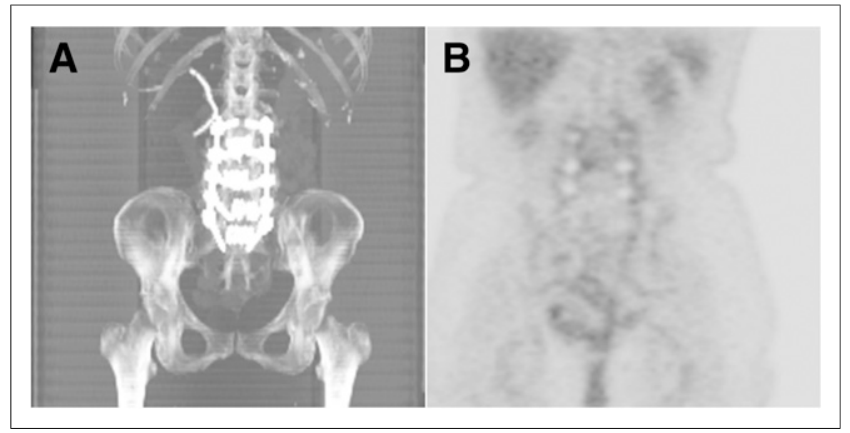

FIGURE 4. Uninfected spinal hardware. (A) ${ }^{18} \mathrm{~F}-\mathrm{FDG}$ PET/CT scout radiograph demonstrates lumbar spine hardware. (B) Coronal ${ }^{18} \mathrm{~F}-\mathrm{FDG}$ image reveals normal lumbar spine activity. Normal ${ }^{18} \mathrm{~F}-\mathrm{FDG}$ findings effectively exclude infection.

\section{Diabetic Pedal Osteomyelitis}

Diabetic patients can have a significant foot infection without a systemic response, and the diagnosis of osteomyelitis often is overlooked (34). Labeled leukocyte imaging is the radionuclide gold standard for diagnosing diabetic pedal osteomyelitis. The sensitivity and specificity of planar ${ }^{111}$ In-labeled leukocyte imaging range from $72 \%$ to $100 \%$ and from $67 \%$ to $100 \%$, respectively. The sensitivity and specificity of planar ${ }^{99 \mathrm{~m} T c-l a b e l e d ~ l e u k o c y t e}$ imaging range from $86 \%$ to $93 \%$ and from $80 \%$ to $98 \%$, respectively (3). Results using radiolabeled antigranulocyte antibodies and antibody fragments are similar (35-37).

SPECT/CT is useful in suspected pedal osteomyelitis (38-42). Heiba et al. (39) found that simultaneous dual-isotope ${ }^{111}$ In-labeled leukocyte/99m Tc-methyl diphosphonate SPECT/CT and marrow imaging were significantly more accurate than planar imaging and single-isotope SPECT/CT, facilitating precise labeled leukocyte localization and improving interpreter confidence. In another investigation, dual-isotope SPECT/CT was more accurate than conventional imaging for diagnosing and localizing infection, helped guide patient management, and was associated with a shorter hospital stay (40).

Filippi et al. (41) reported that ${ }^{99 \mathrm{~m} T \mathrm{~T}-\text {-labeled leukocyte SPECT/ }}$ CT changed study interpretation in more than half the cases, confirming or excluding osteomyelitis and precisely defining the extent of infection (Fig. 5). Erdman et al. (42) developed the Composite Severity Index for ${ }^{99 \mathrm{~m}}$ Tc-labeled leukocyte SPECT/CT. The likelihood of a favorable outcome varied inversely with the Composite Severity Index score, which predicted outcome more accurately than did classifying studies as positive or negative for osteomyelitis.

Vouillarmet et al. (43) used ${ }^{99 \mathrm{~m}}$ Tc-labeled leukocyte SPECT/CT to monitor the treatment response in diabetics with pedal osteomyelitis. The test was negative in 22 patients and positive in 7 patients, including 5 who subsequently relapsed. Sensitivity, specificity, positive predictive value, and negative predictive value for osteomyelitis relapse were $100 \%, 91.5 \%, 71.5 \%$, and $100 \%$. Lazaga et al. (44) reported that ${ }^{99 \mathrm{~m} T c-l a b e l e d ~ l e u k o c y t e ~ S P E C T / C T ~ w a s ~} 90 \%$ sensitive and $56 \%$ specific for determining treatment response.

Although most investigations involve labeled leukocyte imaging, Aslangul et al. (45) reported that ${ }^{67} \mathrm{Ga}$ SPECT/CT was $88 \%$ sensitive and $93.6 \%$ specific for diabetic pedal osteomyelitis.

Several groups have investigated ${ }^{18} \mathrm{~F}-\mathrm{FDG}$ in diabetic foot infections (46-53). Basu et al. (46) reported that ${ }^{18} \mathrm{~F}-\mathrm{FDG}$ PET was $94 \%$ accurate for differentiating osteomyelitis and soft-tissue infection from the neuropathic joint. Nawaz et al. (47) reported that ${ }^{18}$ F-FDG PET was $81 \%$ accurate for pedal osteomyelitis. Kagna et al. (49) reported that ${ }^{18} \mathrm{~F}$-FDG PET/CT was $96 \%$ accurate for pedal osteomyelitis.

Yang et al. (50) reported that ${ }^{18} \mathrm{~F}$-FDG PET was $93.8 \%$ accurate for pedal osteomyelitis. Sensitivity was nearly identical for patients with serum glucose levels above and below $150 \mathrm{mg} / \mathrm{dL}$ : $88.9 \%$ and $88.3 \%$, respectively. The investigators concluded that mildly to moderately elevated serum glucose levels do not adversely affect sensitivity.

Shagos et al. (51) reported that ${ }^{18}$ F-FDG PET was more specific than bone scintigraphy for osteomyelitis, whereas bone scintigraphy was more sensitive than ${ }^{18} \mathrm{~F}$-FDG PET for the neuropathic joint. Schwegler et al. (52). reported that ${ }^{18}$ F-FDG PET detected 2 of 7 cases (29\% sensitivity) of osteomyelitis. They speculated that low sensitivity may have been related to decreased inflammatory response in the study population or impaired bony uptake of ${ }^{18} \mathrm{~F}-\mathrm{FDG}$, because of insulin resistance. Motion artifacts and limited spatial resolution also may have contributed to low sensitivity.

Familiari et al. (53) compared ${ }^{18} \mathrm{~F}$-FDG PET/CT with planar ${ }^{99 m}$ Tc-labeled leukocyte imaging. ${ }^{18} \mathrm{~F}-\mathrm{FDG}$ PET/CT accuracy was 


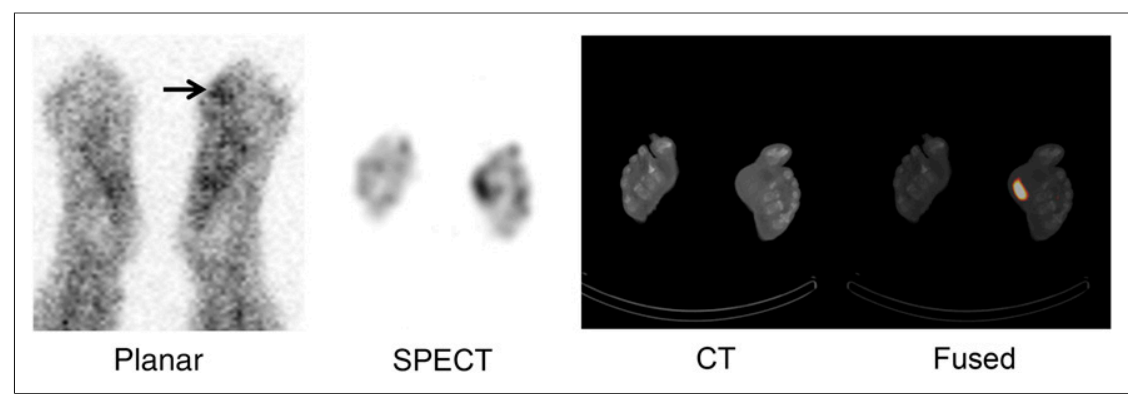

FIGURE 5. Soft-tissue infection of left foot. Increased activity (arrow) underlies left first metatarsal on planar ${ }^{99 \mathrm{~m} T c}$-labeled leukocyte image. There is no way to determine whether this focus extends into bone. Coronal SPECT/CT localizes infection to soft tissue. By precisely localizing areas of radiopharmaceutical uptake, SPECT/CT can guide patient management.

$54 \%$, improving to $62 \%$ when CT findings were included. The accuracy of planar ${ }^{99 \mathrm{~m}} \mathrm{Tc}$-labeled leukocyte imaging was $92 \%$.

The role of ${ }^{18} \mathrm{~F}-\mathrm{FDG}$ for diagnosing diabetic pedal osteomyelitis is uncertain. Limitations of available data include varying methodology, different patient populations, inconsistent correlation with MRI, absence of a uniform truth standard, and few comparative studies with labeled leukocyte imaging $(54,55)$. Treglia et al. (55), in a metaanalysis, reported a sensitivity and specificity of $74 \%$ and $91 \%$ for ${ }^{18} \mathrm{~F}$-FDG. They concluded that large, multicenter studies using bone biopsy as the gold standard are warranted.

\section{Prosthetic Joint Infection}

Aseptic loosening, the most common cause of prosthetic joint failure, frequently results from an immune response by the patient's body against one or more of the prosthetic components. The immune response can be accompanied by an intense inflammatory response involving large numbers of leukocytes. Aseptic loosening usually is managed with a single-stage exchange arthroplasty requiring one hospital admission and surgical intervention (56).

Infection occurs in $1 \%-2 \%$ of primary implants and up to $5 \%$ of revision implants. Approximately one third of these infections develop within 3 mo (early), another third within 1 y (delayed), and the remainder more than $1 \mathrm{y}$ (late) after surgery (56). The inflammatory reaction accompanying infection can be similar to that in aseptic loosening, except that neutrophils, usually absent in aseptic loosening, invariably are present in large numbers in infection. Treatment consists of excisional arthroplasty followed by antibiotics and, eventually, revision arthroplasty (56).

Differentiating aseptic loosening from infection, important because their treatments are very different, can be difficult. Signs

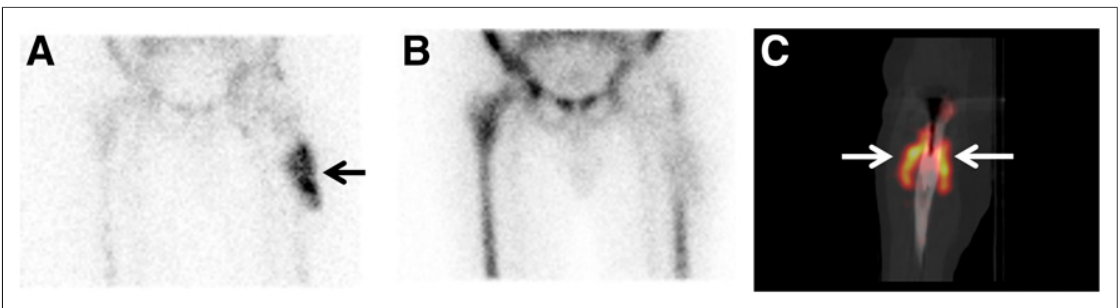

FIGURE 6. Infected left-hip arthroplasty. (A and B) There is intense activity along lateral aspect of femoral component of prosthesis (arrow) on anterior ${ }^{111} \mathrm{In}$-labeled leukocyte image (A), with no corresponding activity on ${ }^{99 \mathrm{mT}} \mathrm{T}$-sulfur colloid bone marrow image (B). Study confirms infection but provides little information about extent. (C) On sagittal SPECT/CT image, infection extends anteriorly and posteriorly into soft tissues surrounding prosthesis (arrows). This information is useful for surgical planning. of infection may be absent. Abnormal laboratory values are suggestive, but not diagnostic, of infection. Joint aspiration with culture is specific; sensitivity, however, is variable (56). Plain radiographs lack specificity. Hardware-induced artifacts limit, to some degree, cross-sectional imaging.

Radionuclide imaging has a preeminent position in the evaluation of joint arthroplasty infection. The bone scan has an accuracy of $50 \%-70 \%$, which does not improve when performed as a 3-phase study (56). Combined bone $/{ }^{67} \mathrm{Ga}$ scintigraphy, with an accuracy of $60 \%-80 \%$, offers only a modest improvement over bone scintigraphy alone (56). Labeled leukocyte/marrow imaging, with an accuracy of about $90 \%$, currently is the best imaging test available. All the published studies confirm high specificity; nearly all also indicate high sensitivity (56).

The sensitivity and specificity of $99 \mathrm{~m} \mathrm{Tc}$-besilesomab for joint replacement infection range from $67 \%$ to $91 \%$ and from $57 \%$ to $75 \%$, respectively. Complementary bone imaging and semiquantitative analysis improve accuracy (56).

The sensitivity and specificity of $99 \mathrm{~m} \mathrm{Tc}$-sulesomab range from $75 \%$ to $93 \%$ and from $65 \%$ to $86 \%$, respectively. Dual-time-point imaging, time-activity curve analysis, and complementary marrow imaging improve accuracy (56).

Data on SPECT/CT in prosthetic joint infection are encouraging (57-62). Tam et al. (57) reported that the CT component of bone SPECT/CT identifies morphologic abnormalities that correspond to areas of increased activity on radionuclide images. Joint distension, fluid-filled bursa, and intramuscular fluid collections, findings that are sensitive and specific for infection, can be identified on the CT component of the examination. Al-Nabhani et al. (58) reported that bone SPECT/CT contributed useful information in more than $80 \%$ of patients with a painful knee arthroplasty. Filippi et al. (59) reported that the accuracy of ${ }^{99 \mathrm{~m} T c-l a b e l e d ~ l e u k o c y t e ~ i m a g i n g ~ i m p r o v e d ~ f r o m ~} 64 \%$ for scintigraphy with SPECT to $100 \%$ for SPECT/CT. SPECT/CT precisely localized labeled leukocyte accumulation, facilitating the differentiation of soft-tissue from bone infection. Kim et al. (60) reported sensitivity, specificity, and accuracy of $82.0 \%, 88.0 \%$, and $84.8 \%$, respectively, for planar imaging. Sensitivity, specificity, and accuracy increased to $93.3 \%$ with SPECT/CT. SPECT/CT precisely localized the site, and accurately delineated the extent, of infection (Fig. 6).

Graute et al. (62) reported sensitivity, specificity, and accuracy of $66 \%, 60 \%$, and $61 \%$, respectively, for ${ }^{99 \mathrm{~m} T c-b e s i l e s o m a b}$ planar imaging. By adding SPECT/CT, sensitivity, specificity, and accuracy improved to $77 \%$, $89 \%$, and $73 \%$, respectively.

The results of ${ }^{18} \mathrm{~F}-\mathrm{FDG}$ for diagnosing prosthetic joint infection have varied. Good results have been reported by some investigators. Zhuang et al. (63) reported that ${ }^{18}$ F-FDG PET was $89.5 \%$ and $77.8 \%$ accurate for hip and knee arthroplasty infection, respectively. Correct diagnosis depended on location, not intensity, of uptake. Reinartz et al. (64) reported that ${ }^{18}$ F-FDG PET was 95\% accurate for hip arthroplasty infection. Basu et al. (65) reported sensitivity and specificity of $81.8 \%$ and $93.1 \%$, respectively, for hip 
arthroplasty infection and $94.7 \%$ and $88.2 \%$, respectively, for knee arthroplasty infection. Other investigators reported similar results (6668). Some investigators reported that ${ }^{18} \mathrm{~F}$-FDG uptake around the femoral head and neck is not specific for infection; others reported that this pattern indicates synovitis plus infection $(56,69,70)$.

The results of other investigations have been less satisfactory (71-77). Van Acker et al. reported $100 \%$ sensitivity and $73 \%$ specificity for prosthetic knee infection (71). Stumpe et al. reported that ${ }^{18}$ F-FDG PET was $69 \%$ accurate for prosthetic hip infection (73) and that periprosthetic ${ }^{18} \mathrm{~F}-\mathrm{FDG}$ accumulation around a knee arthroplasty was not specific for infection (74). Delank et al. (75) concluded that ${ }^{18} \mathrm{~F}$-FDG PET was not specific for lower-extremity joint arthroplasty infection. Another group of investigators found that the test was neither sensitive (64\%) nor specific (67\%) for prosthetic hip infection (76). Love et al. (77) reported that ${ }^{18}$ F-FDG was $71 \%$ accurate for lower-extremity prosthetic joint infection.

Comparative investigations of ${ }^{18} \mathrm{~F}-\mathrm{FDG}$ and bone or labeled leukocyte imaging have been contradictory. Some investigations indicated that ${ }^{18} \mathrm{~F}$-FDG is more accurate than bone scintigraphy; others suggested the opposite $(64,71,73)$. Pill et al. (78) reported that ${ }^{18}$ F-FDG PET was $95 \%$ sensitive and $93 \%$ specific for infection. In a subgroup, the sensitivity and specificity of labeled leukocyte/marrow imaging were $50 \%$ and $95.1 \%$, respectively. Love et al. (77) found that labeled leukocyte/marrow imaging was more accurate than ${ }^{18}$ F-FDG (95\% vs. $71 \%$ ). Basu et al. (65) compared ${ }^{18}$ F-FDG PET to labeled leukocyte/marrow imaging in 88 lower-extremity arthroplasties (59 hips, 29 knees). Although their specificities were very similar, ${ }^{18} \mathrm{~F}$-FDG PET was significantly more sensitive than labeled leukocyte/marrow imaging (76.9\% vs. $38.5 \%$ ) for hip prosthesis infection. All 3 infected knee arthroplasties were positive on ${ }^{18} \mathrm{~F}-\mathrm{FDG}$ PET, versus only 1 of 3 on labeled leukocyte/marrow imaging.

In a recent metaanalysis, the pooled sensitivity and specificity of ${ }^{18} \mathrm{~F}$-FDG PET and PET/CT for lower-extremity prosthetic joint infection both were $86 \%$ (79).

The development of an infection-specific imaging agent would be a substantial improvement over currently available radiopharmaceuticals. Aryana et al. (80) reported that ${ }^{99 \mathrm{~m}} \mathrm{Tc}$-ubiquicidin 29-41 was $100 \%$ accurate for hip arthroplasty infection. Though encouraging, these results must be confirmed in larger series.

\section{CONCLUSION}

Radionuclide imaging continues to play a vital role in the diagnostic workup of patients suspected of having musculoskeletal infection. The roles of bone and ${ }^{67} \mathrm{Ga}$ imaging have decreased over time because they have been replaced by more appropriate procedures such as labeled leukocyte imaging and, in the case of spinal infections, ${ }^{18} \mathrm{~F}-\mathrm{FDG}$ imaging. The hybrid imaging techniques SPECT/CT and PET/CT are redefining the role of radionuclide imaging in the management of patients with suspected or known musculoskeletal infection not only by improving diagnostic accuracy but also by demonstrating the potential of radiopharmaceuticals to monitor response to treatment. Although the currently available agents reflect host response, infectionspecific radiopharmaceuticals, such as radiolabeled peptides, show promise both for diagnosis and for monitoring response to treatment.

\section{REFERENCES}

1. Palestro CJ. Nuclear medicine imaging of osteomyelitis: white blood cell, monoclonal antibody, or bacterial imaging? In: Signore A, Quintero AM, eds. Diagnostic Imaging of Infections and Inflammatory Diseases: A Multidisciplinary Approach. New York, NY: John Wiley \& Sons; 2013:168-186.
2. Palestro CJ, Love C, Miller TT. Imaging of musculoskeletal infections. Best Pract Res Clin Rheumatol. 2006;20:1197-1218.

3. Palestro CJ. Radionuclide imaging of osteomyelitis. Semin Nucl Med. 2015; 45:32-46.

4. Palestro CJ, Love C, Bhargava KK. Labeled leukocyte imaging: current status and future directions. $Q \mathrm{~J}$ Nucl Med Mol Imaging. 2009;53:105-123.

5. Palestro CJ, Love C, Tronco GG, Tomas MB, Rini JN. Combined labeled leukocyte and technetium-99m sulfur colloid marrow imaging for diagnosing musculoskeletal infection. Radiographics. 2006;26:859-870.

6. Glaudemans AW, De Vries EF, Vermeulen LE, Slart RH, Dierckx RA, Signore A. A large retrospective single centre study to define best image acquisition protocols and interpretation criteria for white blood cell scintigraphy with ${ }^{99 m}$ Tc-HMPAO labelled leukocytes in musculoskeletal infections. Eur J Nucl Med Mol Imaging. 2013;40:1760-1769.

7. Palestro CJ, Glaudemans AWJM, Dierckx RAJO. Multiagent imaging of inflammation and infection with radionuclides. Clin Transl Imaging. 2013;1:385-396.

8. Kennedy DO. B vitamins and the brain: mechanisms, dose and efficacy - a review. Nutrients. 2016;8:68.

9. Wang G. Human antimicrobial peptides and proteins. Pharmaceuticals (Basel). 2014;7:545-594.

10. Hiemstra PS, van den Barselaar MT, Roest M, Nibbering PH, van Furth R. Ubiquicidin, a novel murine microbicidal protein present in the cytosolic fraction of macrophages. Leukoc Biol. 1999;66:423-428.

11. Vilche M, Reyes AL, Vasilskis E, Oliver P, Balter HS, Engler HW. ${ }^{68}$ Ga-NOTA-UBI $29-$ 41 as a PET tracer for detection of bacterial infection. J Nucl Med. 2016;57:622-627.

12. Kumar V, Boddeti DK. ${ }^{68} \mathrm{Ga}$-radiopharmaceuticals for PET imaging of infection and inflammation. Recent Results Cancer Res. 2013;194:189-219.

13. Diaz LA, Foss CA, Thornton K, et al. Imaging of musculoskeletal bacterial infections by [124I]FIAU-PET/CT. PLoS One. 2007;2:e1007.

14. [ $\left.{ }^{124} \mathrm{I}\right]$ FIAU-PET/CT scanning in patients with pain in a prosthetic knee or hip joint (PJI). ClinicalTrials.gov website. https://clinicaltrials.gov/ct2/show/NCT01705496. Published October 10, 2012. Updated February 8, 2016. Accessed July 12, 2016.

15. FIAU-PET/CT scanning in diagnosing osteomyelitis in patients with diabetic foot infection. https://clinicaltrials.gov/ct2/show/NCT01764919. Published January 7, 2013. Updated April 6, 2016. Accessed July 12, 2016.

16. Gouliouris T, Aliyu SH, Brown NM. Spondylodiscitis: update on diagnosis and management. J Antimicrob Chemother. 2010;65(suppl 3):iii11-iii24.

17. Love C, Patel M, Lonner BS, Tomas MB, Palestro CJ. Diagnosing spinal osteomyelitis: a comparison of bone and gallium scintigraphy and magnetic resonance imaging. Clin Nucl Med. 2000;25:963-977.

18. Gemmel F, Dumarey N, Palestro CJ. Radionuclide imaging of spinal infections. Eur J Nucl Med Mol Imaging. 2006;33:1226-1237.

19. Lazzeri E, Pauwels EKJ, Erba P, et al. Clinical feasibility of two-step streptavidin/111 Inbiotin scintigraphy in patients with suspected vertebral osteomyelitis. Eur J Nucl Med Mol Imaging. 2004;31:1505-1511.

20. Lazzeri E, Erba P, Perri M, Doria R, Tascini C, Mariani G. Clinical impact of SPECT/CT with In-111 biotin on the management of patients with suspected spine infection. Clin Nucl Med. 2010;35:12-17.

21. Guhlmann A, Brecht-Krauss D, Suger G, et al. Fluorine-18-FDG PET and technetium-99m antigranulocyte antibody scintigraphy in chronic osteomyelitis. $J$ Nucl Med. 1998;39:2145-2152.

22. Gratz S, Dorner J, Fischer U, et al. F-18-FDG hybrid PET in patients with suspected spondylitis. Eur J Nucl Med Mol Imaging. 2002;29:516-524.

23. Fuster $\mathrm{D}$, Solà $\mathrm{O}$, Soriano $\mathrm{A}$, et al. A prospective study comparing whole-body FDG PET/CT to combined planar bone scan with ${ }^{67} \mathrm{Ga}$ SPECT/CT in the diagnosis of spondylodiskitis. Clin Nucl Med. 2012;37:827-832.

24. Ohtori S, Suzuki M, Koshi T, et al. ${ }^{18}$ F-fluorodeoxyglucose-PET for patients with suspected spondylitis showing Modic change. Spine. 2010;35:E1599-E1603.

25. Stumpe KD, Zanetti M, Weishaupt D, Hodler J, Boos N, Von Schulthess GK. FDG positron emission tomography for differentiation of degenerative and infectious endplate abnormalities in the lumbar spine detected on MR imaging. AJR. 2002;179:1151-1157.

26. Seifen T, Rettenbacher L, Thaler C, Holzmannhofer J, Mc Coy M, Pirich C. Prolonged back pain attributed to suspected spondylodiscitis: the value of ${ }^{18} \mathrm{~F}-\mathrm{FDG}$ PET/ CT imaging in the diagnostic work-up of patients. Nuklearmedizin. 2012;51:194-200.

27. Fuster D, Tomás X, Mayoral M, et al. Prospective comparison of whole-body ${ }^{18} \mathrm{~F}-\mathrm{FDG} \mathrm{PET} / \mathrm{CT}$ and MRI of the spine in the diagnosis of haematogenous spondylodiscitis. Eur J Nucl Med Mol Imaging. 2015;42:264-271.

28. Skanjeti A, Penna D, Douroukas A, et al. PET in the clinical work-up of patients with spondylodiscitis: a new tool for the clinician? $Q \mathrm{~J}$ Nucl Med Mol Imaging. 2012;56:569-576.

29. Ioannou S, Chatziioannou S, Pneumaticos SG, Zormpala A, Sipsas NV. Fluorine18 fluoro-2-deoxy-D-glucose positron emission tomography/computed tomography scan contributes to the diagnosis and management of brucellar spondylodiskitis. BMC Infect Dis. 2013;13:73. 
30. Nakahara M, Ito M, Hattori N, et al. ${ }^{18} \mathrm{~F}-\mathrm{FDG}-\mathrm{PET} / \mathrm{CT}$ better localizes active spinal infection than MRI for successful minimally invasive surgery. Acta Radiol. 2015;56:829-836.

31. Riccio SA, Chu AKM, Rabin HR, Kloiber R. Fluorodeoxyglucose positron emission tomography/computed tomography interpretation criteria for assessment of antibiotic treatment response in pyogenic spine infection. Can Assoc Radiol J. 2015;66:145-152.

32. De Winter F, Gemmel F, Van de Wiele C, Poffijn B, Uyttendaele D, Dierckx R. 18-fluorine fluorodeoxyglucose positron emission tomography for the diagnosis of infection in the postoperative spine. Spine. 2003;28:1314-1319.

33. Nanni C, Errani C, Boriani L, et al. ${ }^{68} \mathrm{Ga}$-citrate PET/CT for evaluating patients with infections of the bone: preliminary results. J Nucl Med. 2010;51:1932-1936.

34. Palestro CJ, Love C. Nuclear medicine and diabetic foot infections. Semin Nucl Med. 2009;39:52-65.

35. Dominguez-Gadea L, Martin-Curto LM, de la Calle H, Crespo A. Diabetic foot infections: scintigraphic evaluation with 99Tcm-labelled anti-granulocyte antibodies. Nucl Med Commun. 1993;14:212-218.

36. Harwood SJ, Valdivia S, Hung GL, Quenzer RW. Use of sulesomab, a radiolabeled antibody fragment, to detect osteomyelitis in diabetic patients with foot ulcers by leukoscintigraphy. Clin Infect Dis. 1999;28:1200-1205.

37. Delcourt A, Huglo D, Prangere T, et al. Comparison between Leukoscan ${ }^{\circledR}$ (sulesomab) and gallium-67 for the diagnosis of osteomyelitis in the diabetic foot. Diabetes Metab. 2005;31:125-133.

38. Przybylski MM, Holloway S, Vyce SD, Obando A. Diagnosing osteomyelitis in the diabetic foot: a pilot study to examine the sensitivity and specificity of $\mathrm{Tc}^{99 \mathrm{~m}}$ white blood cell-labelled single photon emission computed tomography/ computed tomography. Int Wound J. 2016;13:382-389.

39. Heiba SI, Kolker D, Mocherla B, et al. The optimized evaluation of diabetic foot infection by dual isotope SPECT/CT imaging protocol. J Foot Ankle Surg. 2010;49:529-536.

40. Heiba S, Kolker D, Ong L, et al. Dual-isotope SPECT/CT impact on hospitalized patients with suspected diabetic foot infection: saving limbs, lives, and resources. Nucl Med Commun. 2013;34:877-884.

41. Filippi L, Uccioli L, Giurato L, Schillaci O. Diabetic foot infection: usefulness of SPECT/CT for ${ }^{99 m}$ Tc-HMPAO-labeled leukocyte imaging. J Nucl Med. 2009;50:1042-1046.

42. Erdman WA, Buethe J, Bhore R, et al. Indexing severity of diabetic foot infection with ${ }^{99 m}$ Tc-WBC SPECT/CT hybrid imaging. Diabetes Care. 2012;35:1826-1831.

43. Vouillarmet J, Morelec I, Thivolet C. Assessing diabetic foot osteomyelitis remission with white blood cell SPECT/CT imaging. Diabet Med. 2014;31:1093-1099.

44. Lazaga F, Van Asten SA, Nichols A, et al. Hybrid imaging with ${ }^{99 \mathrm{~m}} \mathrm{Tc}-\mathrm{WBC}$ SPECT/CT to monitor the effect of therapy in diabetic foot osteomyelitis. Int Wound J. April 8, 2015 [Epub ahead of print].

45. Aslangul E, M'bemba J, Caillat-Vigneron N, et al. Diagnosing diabetic foot osteomyelitis in patients without signs of soft tissue infection by coupling hybrid ${ }^{67} \mathrm{Ga}$ SPECT/ CT with bedside percutaneous bone puncture. Diabetes Care. 2013;36:2203-2210.

46. Basu S, Chryssikos T, Houseni M, et al. Potential role of FDG-PET in the setting of diabetic neuro-osteoarthropathy: can it differentiate uncomplicated Charcot's neuropathy from osteomyelitis and soft tissue infection? Nucl Med Commun. 2007;28:465-472.

47. Nawaz A, Torigian DA, Siegelman ES, Basu S, Chryssikos T, Alavi A. Diagnostic performance of FDG-PET, MRI, and plain film radiography (PFR) for the diagnosis of osteomyelitis in the diabetic foot. Mol Imaging Biol. 2010;12:335-342.

48. Keidar Z, Militianu D, Melamed E, Bar-Shalom R, Israel O. The diabetic foot: initial experience with ${ }^{18} \mathrm{~F}$-FDG-PET/CT. J Nucl Med. 2005;46:444-449.

49. Kagna O, Srour S, Melamed E, Militianu D, Keidar Z. FDG PET/CT imaging in the diagnosis of osteomyelitis in the diabetic foot. Eur J Nucl Med Mol Imaging. 2012;39:1545-1550.

50. Yang H, Zhuang H, Rubello D, Alavi A. Mild-to-moderate hyperglycemia will not decrease the sensitivity of ${ }^{18} \mathrm{~F}$-FDG PET imaging in the detection of pedal osteomyelitis in diabetic patients. Nucl Med Commun. 2016;37:259-262.

51. Shagos GS, Shanmugasundaram P, Varma AK, Padma S, Sarma M. 18-F fluorodeoxy glucose positron emission tomography-computed tomography imaging: a viable alternative to three phase bone scan in evaluating diabetic foot complications? Indian J Nucl Med. 2015;30:97-103.

52. Schwegler B, Stumpe KD, Weishaupt D, et al. Unsuspected osteomyelitis is frequent in persistent diabetic foot ulcer and better diagnosed by MRI than by ${ }^{18}$ F-FDG PET or ${ }^{99 m}$ Tc-MOAB. J Intern Med. 2008;263:99-106.

53. Familiari D, Glaudemans AWJM, Vitale V, et al. Can sequential ${ }^{18} \mathrm{~F}-\mathrm{FDG}-\mathrm{PET} / \mathrm{CT}$ imaging replace WBC imaging in the diabetic foot? J Nucl Med. 2011;52:1012-1019.

54. Palestro CJ. FDG and diabetic foot infections: the verdict is... J Nucl Med. 2011;52:1009-1011.

55. Treglia G, Sadeghib R, Annunziata S, et al. Diagnostic performance of fluorine18-fluorodeoxyglucose positron emission tomography for the diagnosis of osteomyelitis related to diabetic foot: a systematic review and a meta-analysis. Foot (Edinb). 2013;23:140-148.
56. Palestro CJ. Nuclear medicine and the failed joint replacement: past, present, and future. World J Radiol. 2014;6:446-458.

57. Tam HH, Bhaludin B, Rahman F, Weller A, Ejindu V, Parthipun A. SPECT-CT in total hip arthroplasty. Clin Radiol. 2014;69:82-95.

58. Al-Nabhani K, Michopoulou S, Allie R. Painful knee prosthesis: can we help with bone SPECT/CT? Nucl Med Commun. 2014;35:182-188.

59. Filippi L, Schillaci O. Usefulness of Tc-99m HMPAO-labeled leukocyte scintigraphy for bone and joint infections. J Nucl Med. 2006;47:1908-1913.

60. Kim HO, Na SJ, Oh SJ, et al. Usefulness of adding SPECT/CT to ${ }^{99 \mathrm{~m}} \mathrm{Tc}-$ hexamethylpropylene amine oxime (HMPAO)-labeled leukocyte imaging for diagnosing prosthetic joint infections. J Comput Assist Tomogr. 2014;38:313-319.

61. Kaisidis A, Megas P, Apostolopoulos D, et al. Diagnosis of septic loosening of hip prosthesis with LeukoScan: SPECT scan with ${ }^{99 \mathrm{~m}} \mathrm{Tc}$-labeled monoclonal antibodies. Orthopade. 2005;34:462-469.

62. Graute V, Feist M, Lehner S, et al. Detection of low-grade prosthetic joint infections using ${ }^{99 \mathrm{~m} T c-a n t i g r a n u l o c y t e ~ S P E C T / C T: ~ i n i t i a l ~ c l i n i c a l ~ r e s u l t s . ~ E u r ~}$ J Nucl Med Mol Imaging. 2010;37:1751-1759.

63. Zhuang H, Duarte PS, Pourdehnad M, et al. The promising role of ${ }^{18} \mathrm{~F}-\mathrm{FDG}$ PET in detecting infected lower limb prosthesis implants. J Nucl Med. 2001;42:44-48

64. Reinartz P, Mumme T, Hermanns B, et al. Radionuclide imaging of the painful hip arthroplasty: positron-emission tomography versus triple-phase bone scanning. J Bone Joint Surg Br. 2005;87:465-470.

65. Basu S, Kwee TC, Saboury B, et al. FDG PET for diagnosing infection in hip and knee prostheses: prospective study in 221 prostheses and subgroup comparison with combined ${ }^{111} \mathrm{In}$-labeled leukocyte/99m Tc-sulfur colloid bone marrow imaging in 88 prostheses. Clin Nucl Med. 2014;39:609-615.

66. Chacko TK, Zhuang H, Stevenson K, Moussavian B, Alavi A. The importance of the location of fluorodeoxyglucose uptake in periprosthetic infection in painful hip prostheses. Nucl Med Commun. 2002;23:851-855.

67. Cremerius U, Mumme T, Reinartz P, Wirtz D, Niethard FU, Büll U. Analysis of ${ }^{18}$ F-FDG uptake patterns in PET for diagnosis of septic and aseptic loosening after total hip arthroplasty. Nuklearmedizin. 2003;42:234-239.

68. Gravius S, Gebhard M, Ackermann D, Büll U, Hermanns-Sachweh B, Mumme T. Analysis of ${ }^{18}$ F-FDG uptake pattern in PET for diagnosis of aseptic loosening versus prosthesis infection after total knee arthroplasty: a prospective pilot study. Nuklearmedizin. 2010;49:115-123.

69. Beslic N, Heber D, Lipp RW, Sonneck-Koenne C, Knoll P, Mirzaei S. Metabolic pattern of asymptomatic hip-prosthesis by ${ }^{18} \mathrm{~F}$-FDG-positron-emission-tomography. Iran J Radiol. 2015;12:e11204.

70. Manthey N, Reinhard P, Moog F, Knesewitsch P, Hahn K, Tatsch K. The use of $\left[{ }^{18} \mathrm{~F}\right]$ fluorodeoxyglucose positron emission tomography to differentiate between synovitis, loosening and infection of hip and knee prostheses. Nucl Med Commun. 2002;23:645-653.

71. Van Acker F, Nuyts J, Maes A, et al. FDG-PET, ${ }^{99} \mathrm{~m}$ tc-HMPAO white blood cell SPET and bone scintigraphy in the evaluation of painful total knee arthroplasties. Eur J Nucl Med. 2001;28:1496-1504

72. Vanquickenborne B, Maes A, Nuyts J, et al. The value of ${ }^{18}$ FDG-PET for the detection of infected hip prosthesis. Eur J Nucl Med Mol Imaging. 2003;30:705-715.

73. Stumpe KD, Nötzli HP, Zanetti M, et al. FDG PET for differentiation of infection and aseptic loosening in total hip replacements: comparison with conventional radiography and three-phase bone scintigraphy. Radiology. 2004;231:333-341.

74. Stumpe KD, Romero J, Ziegler O, et al. The value of FDG-PET in patients with painful total knee arthroplasty. Eur J Nucl Med Mol Imaging. 2006;33:1218-1225.

75. Delank KS, Schmidt M, Michael JW, Dietlein M, Schicha H, Eysel P. The implications of ${ }^{18} \mathrm{~F}$-FDG PET for the diagnosis of endoprosthetic loosening and infection in hip and knee arthroplasty: results from a prospective, blinded study. BMC Musculoskelet Disord. 2006;7:20.

76. García-Barrecheguren E, Rodríguez Fraile M, Toledo Santana G, Valentí Nín JR, Richter Echevarría JA. FDG-PET: a new diagnostic approach in hip prosthetic replacement. Rev Esp Med Nucl. 2007;26:208-220.

77. Love C, Marwin SE, Tomas MB, et al. Diagnosing infection in the failed joint replacement: a comparison of coincidence detection fluorine-18 FDG and indium111-labeled leukocyte/technetium-99m-sulfur colloid marrow imaging. J Nucl Med. 2004;45:1864-1871.

78. Pill SG, Parvizi J, Tang PH, et al. Comparison of fluorodeoxyglucose positron emission tomography and ${ }^{111}$ indium-white blood cell imaging in the diagnosis of periprosthetic infection of the hip. $J$ Arthroplasty. 2006;21:91-97.

79. Jin H, Yuan L, Li C, Kan Y, Hao R, Yang J. Diagnostic performance of FDG PET or PET/CT in prosthetic infection after arthroplasty: a meta-analysis. $Q \mathrm{~J} \mathrm{Nucl}$ Med Mol Imaging. 2014;58:85-93.

80. Aryana K, Hootkani A, Sadeghi R, et al. ${ }^{99 \mathrm{~m}} \mathrm{Tc}$-labeled ubiquicidin scintigraphy: a promising method in hip prosthesis infection diagnosis. Nuklearmedizin. 2012;51:133-139. 